PROCEEDINGS OF THE

AMERICAN MATHEMATICAL SOCIETY

Volume 129, Number 7, Pages 1923-1926

S 0002-9939(00)05898-6

Article electronically published on November 22, 2000

\title{
A USEFUL SEMISTABILITY CRITERION
}

\author{
ALEXANDER SCHMITT
}

(Communicated by Ron Donagi)

\begin{abstract}
We provide a short proof for a semistability criterion which is crucial to the construction of master spaces which has drawn interest in recent research in Geometric Invariant Theory.
\end{abstract}

Among the main objects of study of Geometric Invariant Theory are representations of reductive algebraic groups. Given a reductive algebraic group $G$, defined over a field $k$, a finite dimensional $k$-vector space $W$, and a rational representation $\rho: G \longrightarrow \mathrm{GL}(W)$, one obtains an action of $G$ on $\mathbb{P}(W)$, the space of hyperplanes in $W$, and a representation of $G$ on the algebra $R:=\operatorname{Sym} W$. By the Hilbert-Nagata theorem, the algebra $R^{G}$ of $G$-invariant elements in $R$ is a finitely generated graded subalgebra of $R$. Therefore, $\mathbb{P}(W) / / G:=\operatorname{Proj} R^{G}$ is a projective variety, and there is a rational map $\pi: \mathbb{P}(W) \rightarrow \mathbb{P}(W) / / G$. This is the GIT procedure of forming a quotient. A closed point $[w] \in \mathbb{P}(W)$ is called semistable, if $\pi$ is defined in $[w]$, i.e., if there is a non-constant homogeneous element in $R^{G}$ not vanishing in $[w]$. The set $\mathbb{P}(W)^{s s}$ of semistable points is open, and a point $[w] \in \mathbb{P}(W)$ is called polystable, if $[w]$ is semistable and the $G$-orbit of $[w]$ is closed in $\mathbb{P}(W)^{s s}$. The map $\pi$ identifies the closed points of $\mathbb{P}(W) / / G$ with the set of $G$-orbits of polystable points.

A central task of GIT is now to identify the semistable and polystable points in $\mathbb{P}(W)$. This is usually achieved by the Hilbert-Mumford criterion [1]. However, if $\rho$ is the direct sum of other representations, this might become too difficult. In this note, we will provide a short and elementary proof of a theorem from [2, dealing with this situation. More precisely, let $k$ and $G$ be as before, and $W_{1}, \ldots, W_{s}$ finite dimensional $k$-vector spaces. Suppose we are given representations $\rho_{i}: G \longrightarrow$ $\operatorname{GL}\left(W_{i}\right), i=1, \ldots, s$, with $W=W_{1} \oplus \cdots \oplus W_{s}$ and $\rho=\rho_{1} \oplus \cdots \oplus \rho_{s}$. For any $\underline{\iota}=\left(\iota_{1}, \ldots, \iota_{t}\right)$ with $0<t \leq s, \iota_{1}, \ldots, \iota_{t} \in\{1, \ldots, s\}$, and $\iota_{1}<\cdots<\iota_{t}$, the $\rho_{i}$ 's yield an action $\sigma_{\underline{\iota}}$ of $G$ on $\mathbb{P}_{\underline{\underline{\iota}}}:=\mathbb{P}\left(W_{\iota_{1}}\right) \times \cdots \times \mathbb{P}\left(W_{\iota_{t}}\right)$, and, for any sequence of positive integers $k_{1}, \ldots, k_{t}$, a linearization of $\sigma_{\underline{\iota}}$ in the very ample line bundle $\mathcal{O}\left(k_{1}, \ldots, k_{t}\right)$. The computation of the semistable and polystable points in $\mathbb{P}(W)$ can be reduced to the computation of the semistable and polystable points in the $\mathbb{P}_{\underline{\underline{L}}}$ 's by means of the following theorem.

Theorem. Let $w=\left[w_{1}, \ldots, w_{s}\right]$ be a point in $\mathbb{P}(W)$, with $w_{i}$ denoting its component in $W_{i}^{\vee}, i=1, \ldots, s$. Then $w$ is semistable (polystable), if and only if there are an index $\underline{\iota}=\left(\iota_{1}, \ldots, \iota_{t}\right)$ such that $j \in\left\{\iota_{1}, \ldots, \iota_{t}\right\}$ implies (is equivalent to) $w_{j} \neq 0$ and positive integers $k_{1}, \ldots, k_{t}$ such that the point $\left(\left[w_{\iota_{1}}\right], \ldots,\left[w_{\iota_{t}}\right]\right)$ in $\mathbb{P}_{\underline{\underline{L}}}$ is semistable (polystable) w.r.t. the given linearization of $\sigma_{\underline{\underline{L}}}$ in $\mathcal{O}\left(k_{1}, \ldots, k_{t}\right)$.

Received by the editors November 23, 1998 and, in revised form, November 12, 1999.

1991 Mathematics Subject Classification. Primary 14L10, 14D25, 13A50. 
Remark. As one easily checks, for stable points only the "if"-direction remains true. The theorem remains useful even for the identification of stable points, using their characterisation as polystable points with finite stabilizer.

In 2], this theorem was proved for $s=2$ using properties of $\mathbb{C}^{*}$-actions and actions of products of groups. This approach actually not only yields the above theorem but also has applications to chains of GIT-flips. For the construction of master spaces (see below), this extra information is not necessary and it seems reasonable to have a much easier proof of the result.

Example (The coupling principle of Okonek and Teleman [3]). The setup is as follows: We are given rational representations $\rho_{0}: G \longrightarrow \mathrm{GL}\left(W_{0}\right)$ and $\rho_{\infty}: G \longrightarrow$ $\mathrm{GL}\left(W_{\infty}\right)$ as well as $G$-invariant closed subschemes $Y_{0} \subset \mathbb{P}\left(W_{0}\right)$ and $Y_{\infty} \subset \mathbb{P}\left(W_{\infty}\right)$. A coupling of the GIT problem $\left(Y_{0}, \rho_{0}\right)$ to the GIT problem $\left(Y_{\infty}, \rho_{\infty}\right)$ is a $G$-invariant closed subscheme $Y \subset \mathbb{P}\left(W_{0} \oplus W_{\infty}\right)$ which is also invariant under the $\mathbb{C}^{*}$-action given by $z \cdot\left[w_{0}, w_{\infty}\right]:=\left[w_{0}, z w_{\infty}\right]$, such that $Y \cap \mathbb{P}\left(W_{0}\right)=Y_{0}$ and $Y \cap \mathbb{P}\left(W_{\infty}\right)=Y_{\infty}$. The space $\mathcal{M}:=Y / / G$ is called the master space of the coupling. In case $Y_{0}=Y_{\infty}$, this construction is due to Thaddeus 4]. The space $\mathcal{M}$ can now be exploited to gather information on $\mathcal{M}_{0}:=Y_{0} / / G$ and $\mathcal{M}_{\infty}:=Y_{\infty} / / G$. The full scope of this method and sample applications are discussed in [3]. Our theorem now plays a central role in the description of the scheme $\mathcal{M}$.

Example (Moduli spaces of semistable oriented pairs [2]). Oriented pairs have their origins in non-abelian Seiberg-Witten theory. The moduli space of semistable oriented pairs on surfaces and the above-mentioned coupling principle are key ingredients in the program of Okonek and Teleman for relating Donaldson and SeibergWitten invariants. The same circle of ideas can be applied to oriented pairs on curves, e.g., for another proof of the Verlinde formula [3].

As an illustration, fix a smooth projective curve $C$ and a line bundle $L$ on $C$. An $L$-oriented pair is then a triple $(E, \varepsilon, \varphi)$, consisting of a rank two vector bundle $E$ on $C$, an isomorphism $\varepsilon$ : $\operatorname{det} E \longrightarrow L$, and a global section $\varphi$ of $E$. If $\varphi$ is nontrivial, there is a well defined effective divisor $D$ on $C$ such that $\varphi$ embeds $\mathcal{O}_{C}(D)$ as a subbundle of $E$. The $L$-oriented pair $(E, \varepsilon, \varphi)$ is now semistable, if either $E$ is a semistable vector bundle or $\varphi \neq 0$ and $\operatorname{deg} D \leq \operatorname{deg} E / 2$. The second condition can be easily rephrased in terms of the parameter dependent semistability concept for Bradlow pairs 22. The task is to construct the moduli space of semistable $L$-oriented pairs. Using a Gieseker type construction, one of the main steps is to identify the $\operatorname{SL}(V)$-semistable points in $\mathbb{P}\left(W_{1} \oplus W_{2}\right)$ where $W_{1}:=\operatorname{Hom}\left(\bigwedge^{2} V, U_{1}\right)^{\vee}$ and $W_{2}:=\operatorname{Hom}\left(V, U_{2}\right)^{\vee}, V, U_{1}$, and $U_{2}$ being finite dimensional $k$-vector spaces. The Hilbert-Mumford criterion is not helpful here, mainly because one cannot reduce the problem to certain basic one-parameter subgroups as in the construction of the moduli space of vector bundles or Bradlow pairs. By the theorem, we have to compute the semistable points in $\mathbb{P}\left(W_{1}\right), \mathbb{P}\left(W_{2}\right)$, and $\mathbb{P}\left(W_{1}\right) \times \mathbb{P}\left(W_{2}\right)$ w.r.t. the linearization in $\mathcal{O}\left(k_{1}, k_{2}\right)$ for all $k_{1}, k_{2}>0$. In all these cases, one can safely apply the Hilbert-Mumford criterion. The outcome is that the semistable points correspond to semistable $L$-oriented pairs, using the formulation of semistability in terms of the parameter dependent semistability concept for Bradlow pairs. 


\section{Proof of The Theorem}

We first prove the semistable part. By definition, the point $w$ is semistable if and only if, for some $k>0$, there is an invariant section in $H^{0}(\mathcal{O}(k))=S^{k} W$ which does not vanish in $w$. Now, $S^{k} W=\bigoplus_{k_{1}+\cdots+k_{s}=k} S^{k_{1}} W_{1} \otimes \cdots \otimes S^{k_{s}} W_{s}$, and the representation of $G$ on $S^{k} W$ respects this decomposition, i.e., $\left(S^{k} W\right)^{G}=$ $\bigoplus_{k_{1}+\cdots+k_{s}=k}\left(S^{k_{1}} W_{1} \otimes \cdots \otimes S^{k_{s}} W_{s}\right)^{G}$. Therefore, $w$ is semistable if and only if we find a section in some $\left(S^{k_{\iota_{1}}} W_{\iota_{1}} \otimes \cdots \otimes S^{k_{\iota_{t}}} W_{\iota_{t}}\right)^{G}$ which does not vanish in $w$. This is precisely the assertion of the theorem. Note that we have used only the very definition of semistability. The polystable part will follow easily by means of an induction from the following

Claim. Let $w^{\prime}:=\left(\left[w_{\iota_{1}}, w_{\iota_{2}}\right],\left[w_{\iota_{3}}\right], \ldots,\left[w_{\iota_{t}}\right]\right)$ be a point in $\mathbb{P}\left(W_{\iota_{1}} \oplus W_{\iota_{2}}\right) \times \mathbb{P}_{\left(\iota_{3}, \ldots, \iota_{t}\right)}$. Then $w^{\prime}$ is semistable (polystable) w.r.t. the given linearization in $\mathcal{O}\left(k, k_{3}, \ldots, k_{t}\right)$, if and only if either $\left(\left[w_{\iota_{i}}\right],\left[w_{\iota_{3}}\right], \ldots,\left[w_{\iota_{t}}\right]\right)$ is semistable (polystable) in $\mathbb{P}_{\left(\iota_{i}, \ldots, \iota_{t}\right)}$ w.r.t. the linearization in $\mathcal{O}\left(k, k_{3}, \ldots, k_{t}\right)$ for either $i=1$ (and $\left.w_{\iota_{2}}=0\right)$ or $i=2$ (and $\left.w_{\iota_{1}}=0\right)$, or there are positive natural numbers $n, k_{1}$, and $k_{2}$, such that $k_{1}+k_{2}=n k$ and the point $\left(\left[w_{\iota_{1}}\right],\left[w_{\iota_{2}}\right],\left[w_{\iota_{3}}\right], \ldots,\left[w_{\iota_{t}}\right]\right)$ is semistable (polystable) in $\mathbb{P}_{\left(\iota_{1}, \iota_{2}, \iota_{3}, \ldots, \iota_{t}\right)}$ w.r.t. the linearization in $\mathcal{O}\left(k_{1}, k_{2}, n k_{3}, \ldots, n k_{t}\right)$.

The semistable part is proved as before. First, we suppose that $w^{\prime}$ is polystable. With the Hilbert-Mumford criterion [1, the claim can be rephrased as follows: There exist non-negative rational numbers $\kappa_{1}$ and $\kappa_{2}$, not both zero, with $\kappa_{1}+\kappa_{2}=$ $k$ such that for every one-parameter subgroup $\lambda$ of $G$, we have

$$
\kappa_{1} \mu\left(\left[w_{\iota_{1}}\right], \lambda\right)+\kappa_{2} \mu\left(\left[w_{\iota_{2}}\right], \lambda\right)+\sum_{j=3}^{t} k_{j} \mu\left(\left[w_{\iota_{j}}\right], \lambda\right) \geq 0
$$

and the image of $w^{\prime}$ in the respective $\mathbb{P}_{\underline{\iota}}$ is a fixed point for the action of every one-parameter subgroup $\lambda$ for which equality occurs in (1). By the semistable part of the claim, we already know that we can find $\kappa_{1}$ and $\kappa_{2}$ such that (1) holds for every one-parameter subgroup $\lambda$. There are now two possibilities. The first one is that there are two distinct sets $\left(\kappa_{1}^{i}, \kappa_{2}^{i}\right), i=1,2$, for which (1) holds. But then $\left(\kappa_{1}^{3}, \kappa_{2}^{3}\right)$ with $\kappa_{j}^{3}=\left(\kappa_{j}^{1}+\kappa_{j}^{2}\right) / 2$ will be a third one, $\kappa_{j}^{3}>0, j=1,2$, and it is easy to see that, if for $\left(\kappa_{1}^{3}, \kappa_{2}^{3}\right)$ equality in (11) occurs, then $\mu\left(\left[w_{\iota_{1}}\right], \lambda\right)=\mu\left(\left[w_{\iota_{2}}\right], \lambda\right)$. Let $w^{\prime \prime}:=\left(\left[w_{\iota_{1}}^{\prime \prime}, w_{\iota_{2}}^{\prime \prime}\right],\left[w_{\iota_{3}}^{\prime \prime}, \ldots, w_{\iota_{t}}^{\prime \prime}\right]\right)=\lim _{z \rightarrow \infty} \lambda(z) \cdot w^{\prime}$. The condition $\mu\left(\lambda,\left[w_{\iota_{1}}\right]\right)=$ $\mu\left(\lambda,\left[w_{\iota_{2}}\right]\right)$ implies that both $w_{\iota_{1}}^{\prime \prime}$ and $w_{\iota_{2}}^{\prime \prime}$ are non-zero. Therefore,

$$
\lim _{z \longrightarrow \infty} \lambda(z) \cdot\left(\left[w_{\iota_{1}}\right],\left[w_{\iota_{2}}\right],\left[w_{\iota_{3}}, \ldots, w_{\iota_{t}}\right]\right)=\left(\left[w_{\iota_{1}}^{\prime \prime}\right],\left[w_{\iota_{2}}^{\prime \prime}\right],\left[w_{\iota_{3}}^{\prime \prime}, \ldots, w_{\iota_{t}}^{\prime \prime}\right]\right) .
$$

Since this point is again semistable, the "semistable part" of the proposition implies that $w^{\prime \prime}$ is also semistable. This means $w^{\prime \prime}=w^{\prime}$, because $w^{\prime}$ is assumed to be polystable, and we are done. The second possibility is that there is exactly one such pair $\left(\kappa_{1}, \kappa_{2}\right)$, but this implies that, for all $\nu \geq 0$, all invariant sections of $\mathcal{O}\left(k, k_{3}, \ldots, k_{t}\right)^{\otimes \nu}$ not vanishing in $w^{\prime}$ must lie in $S^{\nu \kappa_{1}} W_{\iota_{1}} \otimes S^{\nu \kappa_{2}} W_{\iota_{2}} \otimes S^{\nu k_{3}} W_{\iota_{3}} \otimes$ $\cdots \otimes S^{\nu k_{t}} W_{\iota_{t}}$, and this obviously implies the claim.

The converse follows easily from the fact that for non-negative rational numbers $\kappa_{1}$ and $\kappa_{2}$ with $\kappa_{1}+\kappa_{2}=k$ and a one-parameter subgroup $\lambda$ one has $\mu\left(w^{\prime}, \lambda\right) \geq$ $\kappa_{1} \mu\left(\left[w_{\iota_{1}}\right], \lambda\right)+\kappa_{2} \mu\left(\left[w_{\iota_{2}}\right], \lambda\right)+\sum_{j=3}^{t} k_{j} \mu\left(\left[w_{\iota_{j}}\right], \lambda\right)$. 


\section{ACKNOWLEDGMENTS}

The author thanks the Emmy-Noether Institute at Bar-Ilan University for support and hospitality during the preparation of the article.

\section{REFERENCES}

[1] D. Mumford, Geometric Invariant Theory, Springer, 1965. MR 35:5451

[2] Ch. Okonek, A. Schmitt, A. Teleman, Master spaces for stable pairs, Topology 38 (1999), 117-39. MR 99h:14010

[3] Ch. Okonek, A. Teleman, Master spaces and the coupling principle: from geometric invariant theory to gauge theory, Commun. Math. Phys. 205 (1999), 437-58. CMP 2000:01

[4] M. Thaddeus, Geometric Invariant Theory and flips, J. $\mathcal{A} \mathcal{M S} 9$ (1996), 691-775. MR 96m:14017

Universität GH Essen, FB6 Mathematik und Informatik, D-45117 Essen, Germany

E-mail address: alexander.schmitt@uni-essen.de 